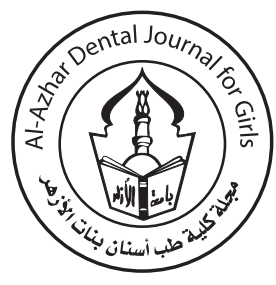

\title{
Assessment the Efficacy of Resin Infiltration in Treatment of Young Permanent Teeth
}

\author{
Somaia A. Ghobar $^{1 *}$, Osama S. El-Shall ${ }^{2}$, Mohamed H. Mustafa ${ }^{3}$
}

Codex : 18/21.04

azhardentj@azhar.edu.eg

http://adjg.journals.ekb.eg

DOI: $10.21608 /$ adjg.2021.23339.1235

Pediatric Dentistry \& Orthodontics ( Pediatric Dentistry, Orthodontics)

\section{KEYWORDS}

Fluoride Varnish, Resin Infiltration, White Spot Lesions

\begin{abstract}
Purpose: To evaluate the efficacy of resin infiltration in treatment of young permanent teeth and to evaluate dental anxiety related to resin infiltration procedure and the time required. Material and methods: 22 Egyptian children ranging from 6-14 years of age were selected with at least 2 smooth surface white lesions (WSL), one on each side using split mouth design. Lesions were assigned in a random way into 2 groups; Group I, Group II. Group I: resin infiltration, Group II: fluoride varnish. Radiographic and Photographic records were taken before treatment, immediately after treatment and 12 months plus assessing the marginal adaptation and discoloration. A facial image scale (FIS) was recorded. The outcome data were recorded, tabulated, coded to be statistically analyzed. Results: by radiographic evaluation immediately after treatment and through the study period the difference was statistically significant in the mean of DSR and RD between groups in favor of group I. By the descriptive analysis the difference of mean of change in both marginal adaptation and discoloration was not a statistically significant. While group I, show increase in mean of marginal adaptation but did not reach to significance level. But within group II, the mean of discoloration has shown statistically significantly difference. Dental anxiety was low in both groups with no statistically significantly difference. Conclusion: Infiltration concept was bridging the gap between "wait and see"; it is a promising micro-invasive approach.
\end{abstract}

- Paper extracted from doctor thesis titled "Assessment the Efficacy of Resin Infiltration in Treatment of Young Permanent Teeth."

1. Assistant Lecturer at Pedodontics and Oral Health, Faculty of Dental Medicine Sinai University, Kantara Campus, Egypt.

2. Professor of Oral Medicine, Periodontology, Oral Diagnosis and Radiology Department Faculty of Dental Medicine for Girls, Al-Azhar University, Cairo, Egypt.

3. Associate Professor, Head of Pedodontics and oral Health Department, Faculty of Dental Medicine for Girls, Al-Azhar University, Cairo, Egypt.

* Corresponding author Email: somia.ghabar@su.edu.eg 


\section{INTRODUCTION}

Dental caries has been one of the commonest childhood diseases as the World Health Organization (WHO) published that it affects about 60 to $90 \%$ of young children. The patterns of dental caries reflect the risk profiles in relation to social level, economic conditions, and application of preventive program. However, it has been founded that dental caries is a reversed disease that can be arrested or reversed if it treated in its early stages ${ }^{(1)}$. According to the principle of minimum intervention, three decades ago, fluoride has been successfully giving good result in caries prevention. It was in the form of dentifrices, gels and solutions which has been effective. Fluoride varnish was developed in the 1960s to lengthen the time of fluoride contact with teeth, since the fluoride release, reactions and uptake depend meanly on the duration of $\operatorname{contact}^{(2-3)}$.

Recently, resin infiltration technique (ICON) accomplished to treat white spot carious lesions. The mean idea of resin infiltration technique, not like sealants that only cover the surface, is to let a low viscosity resin infiltrate to flow into the porous enamel structure, obliterating its tiny pores. Routes by which diffusion of acids and minerals occurred were obliterated in order to arrest the progression of dental caries ${ }^{(2)}$.

The management of dental caries has especial consideration in children treatment to be accepted by child. The response and behavior of children to dental treatment may influenced by the type of dental procedure. Therefore, children self-reporting of their pain and anxiety that accompanied to dental treatment is the most applicable nowadays method, such as facial image scale (FIS) ${ }^{(4-5)}$.

\section{MATERIAL AND METHODS}

\section{Materials used:}

- Resin infiltrant (ICON) (DMG, Hamburg I Germany)

- Fluoride varnish (Flairesse) (DMG Hamburg I Germany)
Case selection: 22 Egyptian children ranging from 6-14 years of age were selected with at least 2 smooth surface white lesions (WSL) detected clinically on the labial surface of permanent anterior teeth, one on each side using split mouth design. The patients were diagnosed in the outpatient Pedodontic Department Clinic, Faculty of Dental Medicine for Girls, Al-Azhar University. Research Ethics Committee approval was obtained from Faculty of Dental Medicine for Girls, Al-Azhar University (REC18-075).Children and their parents or guardians were fully informed about the procedures, possible discomfort, and benefits of this study and asked for agreement (before their participation in this investigation) with a written consent.

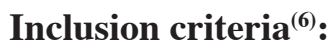

- Children with at least two smooth surface white spot lesions in young permanent teeth.

- Children will be selected in the age range of 6-14 years for better cooperation.

- Inactive white spot lesions having smooth, hard, and shiny surface will be included.

- Children Willing to keep good oral hygiene throughout the study.

- Teeth with score 0, 1,2 according to ICDAS II.

- Absence of preoperative pain, no mobility \& tenderness on percussion.

\section{Exclusion criteria $^{(5-7)}$ :}

- Patients with any medical or dental condition that could impact the study results during its expected length.

- Children who were currently received extensive fluoride regimens.

- Children who show rejection to dental treatment (uncooperative patients).

- Caries lesions showing obvious cavitation, with score 3, 4, 5, 6 according to ICDAS II. 
- Patients who planned to move within 6 months of enrollment.

\section{Methods:}

Patients grouping: The lesions were assigned in a random way into 2 groups according to the applied material: Group I: (22) white spot lesions was Resin Infiltrated by (ICON) according to manufacture direction. Group II: (22) white spot lesions was treated with fluoride varnish (Flairesse) and repeated in the follow up appointment at 3,6 and12 months during the study period. A split mouth design was followed.

Examination of the children: Examination was performed at the beginning of the study period and at follow up for a period of twelve months through the study period. Taking the personal data (name, age as well as detailed medical and dental history) were recorded for each patient, also patients were assessed for their caries risk at the beginning of the study using caries risk assessment form (Age >6) provided by the American dental association 2011 (ADA). According to this form all patients were found to be in the medium and high-risk category. All patients were given verbal oral hygiene instructions to be followed throughout the study. These instructions were: regulating the frequency of taking sugar and sugary drinks, to eat a healthy diet, identical measures of teeth cleaning i.e. brushing teeth 3times a day with fluoridated tooth paste and proper technique of brushing ${ }^{(8)}$.

\section{Assessment tools:}

\section{A. Clinical parameters:}

1. The international caries detection and assessment system scores (ICDAS) ${ }^{(9)}$ : The (ICDAS) was used for clinical examinations and scoring of WSLs; this a visually based system to determine the level of carious lesions at each time point.

2. The discoloration and marginal adaptation tests ${ }^{(10)}$ : Discoloration were recorded according to the following criteria: discoloration of the infiltrated and varnished surfaces was scored as: no discoloration (1), partial discoloration at the margins (2), discoloration of the whole surface (3). The marginal adaptation was recorded as follows: Smooth transition (1) (no detectable margins), Sharp-edged margins (2) (detection with a probe is possible). Patients were clinically examined before treatment and re-examined after 3, 6 and 12 months. The changes in the discoloration and marginal adaptation were assessed.

\section{B. Photographic parameter $^{(11)}$ :}

The photographs have been taken with a digital camera (Sony cyber-shot dsc-W710, Japan with a 9-25mm Sony G lens, 5X optical zoom). The camera setting was adjusted to manual with an aperture of f16; the image fineness was set as ISO sensitivity 200 using macro lens in addition to pro lens zoe camera. Photographic records were performed before and immediately after treatment then in follow up periods the photographs were taken after 3,6 and 12 months in a light controlled environment and camera sited at the same length from the object for standardization.

\section{Radiographic parameter $^{(1)}$ :}

Standardized periapical radiography was used and variation of radio density of lesion was recorded via digital subtraction radiography, manipulating the radiographs recorded at baseline and after treatment. Digital subtraction radiography (DSR): is a software in which we can eliminate the structures out of consideration by storing the pre- and post-operative images in a computer and then combining them together to produce the final subtracted digital image, which represent the net differences. This subtraction measurement was recorded for each selected tooth of each group between base line prior treatment and immediately after treatment then between base line prior treatment and 12 months after treatment. 
Results were calculated, tabulated and statistically analyzed. Assessment criteria (9-10): Standardized reproducible periapical radiographs for each treated tooth were obtained. To achieve such technique, using an XCP anterior film holder, Intra oral sensor size 0. A digital X-ray system (Vista scan Dental Perio, Durr Dental AG, Bietigheim, Germany) and an X-ray machine set at 70Kvp, $8 \mathrm{~mA}$ obtained by a standardized technique and an exposure time of 0.6 seconds. For each patient, information including patient's name, age, radiographic image and all data were recorded and saved in the patient's card on the computer. Results were calculated, tabulated and statistically analyzed.

\section{Patient satisfaction and the required time ${ }^{(12)}$ :}

Facial image scale (FIS) was recorded to each patient after the two different procedure (ICON, Flairesse) and the child was asked to select the best face representing his feelings after the treatment. FIS include five faces, gradually arranged from very happy to very unhappy. Scores 1 and 2 are represented as positive faces, 3 is undefined and 4 and 5 are negative faces (Fig. $1 \mathrm{~h}$ ). The required time for completing the treatment was calculated with stop watch chronometer by an assistant. The stop watch was clicked after application of the Optra gate system ${ }^{(13)}$. Results were calculated, tabulated and statistically analyzed.

\section{Technique of resin infiltration:}

Preparing the patient by scaling and polishing of anterior teeth. Taking pre-operative digital photographs using digital camera and pre-operative digital radiograph using anterior film holder. Isolation by application of Optra gate system then adjust the chronometer. Application or resin according to manufacture direction: 1- Erode the surface with a $15 \% \mathrm{HCL}$ gel; for 2 minutes to be sure that the pores of the lesion were opened. 2Rinsing by water for 30 seconds. 3 - The pore system is then dried with ethanol $99 \%$ for 30 seconds this step gives a view of what the surface will looks like. 4- Drying by oil and water free air for 3 seconds. 5- Application of the resin infiltrate onto the lesion body for 3 minutes. 6- Apply light cure for at least 40 seconds. $7-2^{\text {nd }}$ application of the ICON resin for 1 minute. 8- Repeat light cure for at least 40 seconds. Finally, the labial surface was finished and polished with finishing and polishing kit (Fig.1).

\section{Technique of fluoride varnish application:}

Tooth surfaces were cleaned thoroughly and isolated with Optra gate system. According to manufacturer direction, the unit dose packages were opened, the applicator brush to mix varnish to avoid the separation of fluoride components. A thin coat of varnish was applied. The patient was instructed to close his or her mouth to set the varnish in the presence of saliva and not to rinse or apply suction immediately after application. Post-operative instructions: Children were instructed to avoid brushing, rinsing, eating or drinking for 3-4 hours after treatment, to consume a soft diet and to avoid brushing and flossing for the rest of the day ${ }^{(14)}$. Results were calculated, tabulated and statistically analyzed. (Fig. 1g)

\section{Statistical analysis:}

All collected data were coded and entered to the Statistical Package for Social Science (IBM SPSS) version 23. The data were represented as mean, standard deviations and ranges when their distribution found parametric. Also, qualitative variables were presented as number and percentages. The Independent t-test used to compare between two groups. The paired t- test used to compare between two paired groups with quantitative data and parametric distribution. The repeated ANOVA test used to compare between two more paired groups. By setting the confidence interval to $95 \%$ the margin of error was accepted at $5 \%$. 


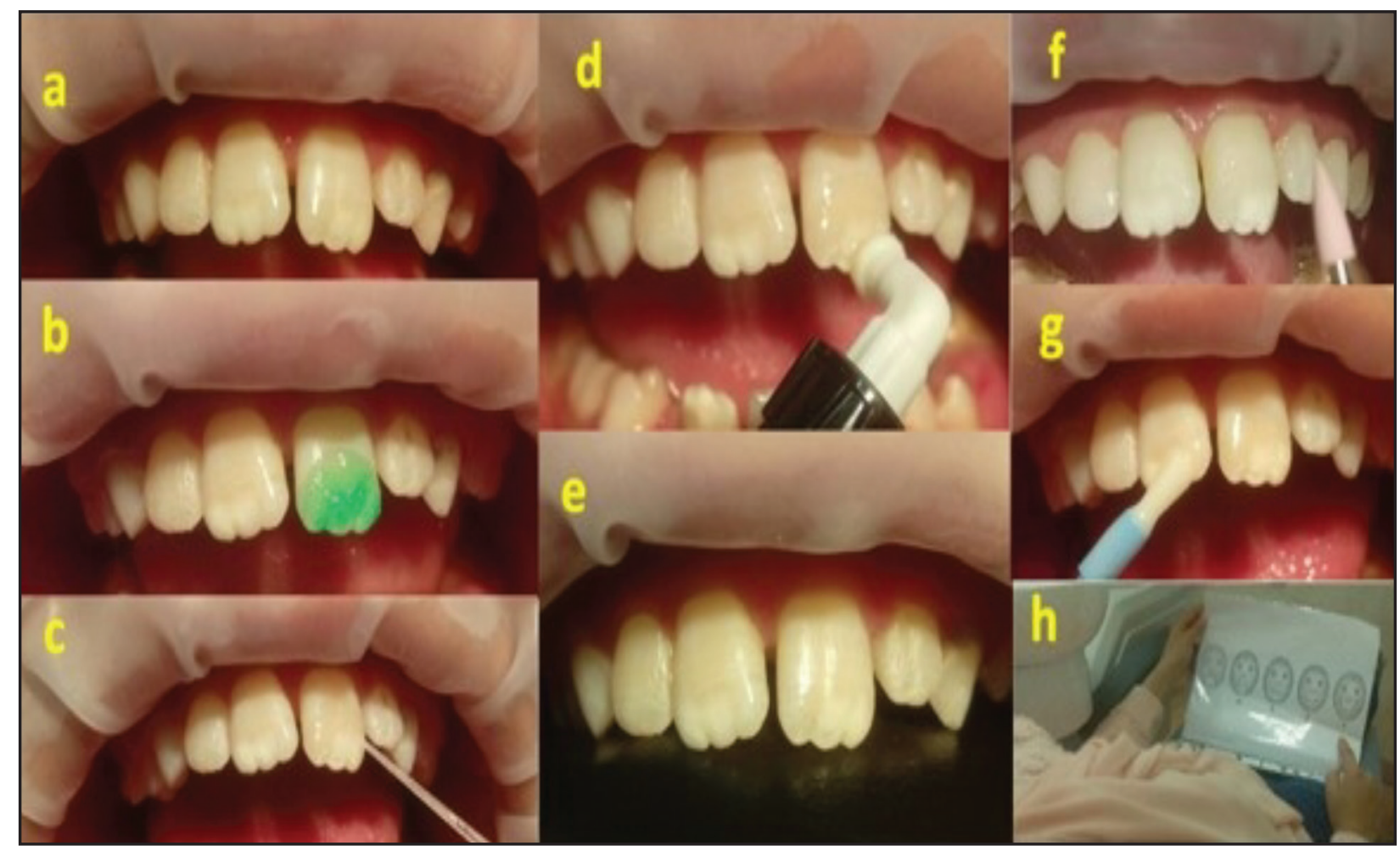

Figure (1): (a,b,c,d) and (e) Representing steps of resin infiltration, (f) Finishing of resin, (g) Application of fluoride varnish, (h) Facial image scale assessment

\section{RESULTS}

\section{Results of clinical evaluation:}

a) ICDAS II: Through all periods, the difference was not statistically significant in the mean of ICDAS II between two groups.

b) Marginal adaptation MA descriptive analysis of mean of change in MA of two groups in the whole study period. At base line, 3 and 6 months, the difference was not statistically significant between the two groups. Moreover, at 12 months, the difference was not statistically significant in the mean of MA of group I. While group I, showed increase in mean of MA but did not reach to significance level. Within group II, the mean of MA had shown statistically significantly increased from the base line to 12 months. c) Discoloration: Descriptive analysis of mean of change in $\mathrm{D}$ of two groups, in the whole study period, the difference was not statistically significant between the two groups. Within the group I, the mean of discoloration had shown no statistically significantly change from the base line value to 12 months (table 1). Within group II, the difference in the mean of discoloration was statistically significant from the base line to 12 months.

\section{Results of radiographic evaluation:}

Immediately after treatment, the difference was statistically significant in the mean of DSR between two groups in favor of group I. Descriptive analysis of mean of change in $\mathrm{RD}$ of two groups in the whole study period. The difference was statistically significant between the two groups in favor of group I at the end of the study table (2). 
Table (1): The mean and SD values and results of comparison in discoloration between the two groups and within each group.

\begin{tabular}{|c|c|c|c|c|c|c|}
\hline \multirow[b]{2}{*}{ Discoloration } & \multicolumn{2}{|c|}{ Group I } & \multicolumn{2}{|c|}{ Group II } & \multirow{2}{*}{ t- Value } & \multirow{2}{*}{ P- Value } \\
\hline & Mean & SD & Mean & SD & & \\
\hline Base line & 1 & 0 & 1 & 0 & 0.000 & 1.000 \\
\hline $3 \mathrm{~m}$ & 1 & 0 & 1 & 0 & 0.000 & 1.000 \\
\hline $6 \mathrm{~m}$ & 1.05 & 0.21 & 1.18 & 0.39 & 0.164 & 1.000 \\
\hline $12 \mathrm{~m}$ & 1.5 & 0.51 & 1.72 & 0.63 & 0.2 & 1.000 \\
\hline \multirow{2}{*}{$\begin{array}{c}\text { Repeated measures } \\
\text { ANOVA }\end{array}$} & $\mathrm{F}$ & 1.26 & 3.376 & & & \\
\hline & P-value & 0.287 & $0.067 *$ & & & \\
\hline
\end{tabular}

Significant difference ( $p$ value $<0.05$ ).

Table (2): The mean of change in RD of two groups in the whole study period.

\begin{tabular}{|c|c|c|c|c|c|c|c|}
\hline \multirow{2}{*}{ RD } & \multicolumn{2}{|c|}{ Before } & \multicolumn{2}{c|}{ Immediate After } & \multicolumn{2}{c|}{ After12months } & \multirow{2}{*}{ P-Value } \\
\cline { 2 - 8 } & Mean & SD & Mean & SD & Mean & SD & \\
\hline Group I & 138.60 & 30.46 & 159.4 & 31.35 & 160.5 & 32.54 & $0.05^{*}$ \\
\hline Group II & 142.5 & 26.79 & 139.6 & 38.45 & 149 & 26.87 & 0.61 \\
\hline
\end{tabular}

Significant difference ( $p$ value $<0.05$ ).

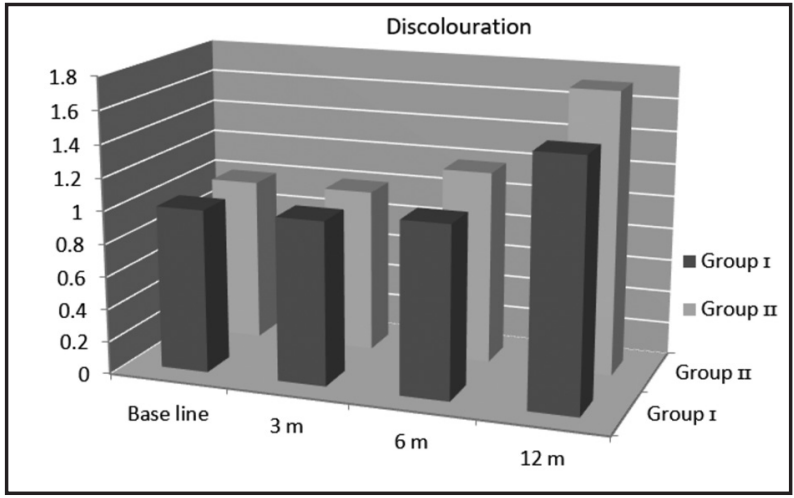

Figure (2): Bar chart representing (mean $\pm \mathrm{SD}$ ) of discoloration between the two groups after treatment.

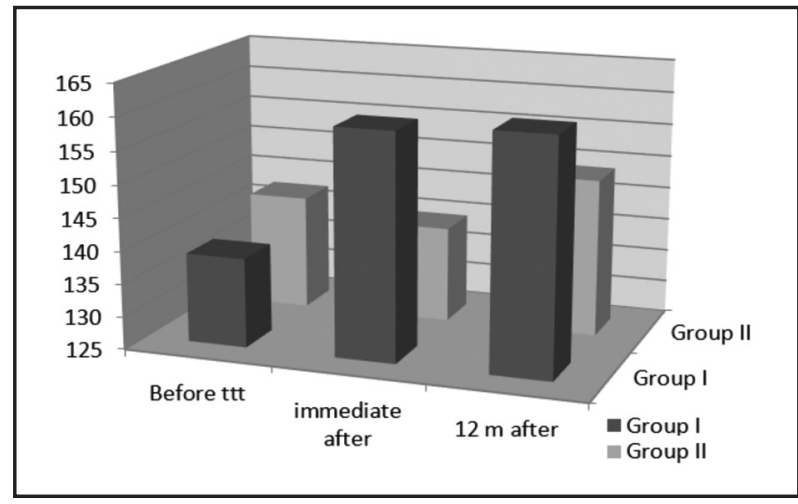

Figure (3): Bar chart representing (mean $\pm \mathrm{SD})$ of RD between the two groups after treatment. 


\section{Results of Patient satisfaction and time required evaluation:}

FIS: Through all periods, the difference in the mean of FIS was not statistically significant between the two groups.

Time: Through all periods, the difference in the mean of time was not statistically significant between the two groups.

\section{DISCUSSION}

Traditional approaches for treating WSLs are application of fluoride representing "wait and see" approach or invasive approach by making restorations. Further dissolution of tooth structure may associate with "Wait and see" approach due to acid diffusion through micro-porosities of incipient caries layer. On the other hand, the invasive approach removes large amount of tooth structure and start "restoration cycle". for these reasons resin Infiltration technique was introduced for bridging the gap between "wait and see" and invasive approaches ${ }^{(8)}$.

Fluoride is the gold standard to which remineralization potential of other remineralizing products could be compared. Fluoride varnish has been used in this study because it can remain in contact with the tooth surface for a long duration by its small amount of high-density, and then penetrate the tooth surface producing bonds ${ }^{(15)}$. Therefore, fluoride varnish was used to increase this contact time. Varnishes are also relatively easy to apply and are well tolerated, making them particularly well suited for children ${ }^{(16)}$. Therefore, the varnish form of fluoride was selected for this study and also select white varnish to be sure not to interfere with esthetic. But over exposure of fluoride can cause fluorosis, especially in young children. That's why, it is important to find other agent free of fluoride ${ }^{(9)}$.

Resin infiltration technique is novel micro invasive approach for management of smooth surface WSLs. The main concept of resin infiltra- tion technique is to use a strong acid to open the micro-pores then obliterate the porosities by a low viscosity, light-cured resin. Unlike sealing, which depend meanly on covering the enamel surface, resin infiltration form diffusion barrier inside the enamel structure. This created barrier add strength to the enamel structure and inhibit its cavitation ${ }^{(17)}$. Therefore, the aim of this study was to evaluate the efficacy of resin infiltration in treatment of young permanent teeth and to evaluate the related dental anxiety and the required time for the treatment.

Some researchers reported that $40 \%$ reduction in the number of WSLs after 3-month follow-up Fluoride varnish application ${ }^{(18)}$. Similarly, another report confirmed that the application of Fluoride varnish was effective in arresting active WSLs, and therefore, reduced the need for restorative intervention ${ }^{(19)}$.In the present study, in Fluoride varnish group, it was found that the RD values of WSLs were significantly increased from baseline to the end of 12 months. These findings were in accordance with previous studies, which reported significant remineralization of early enamel lesions with 5\% sodium Fluoride varnish ${ }^{(2021-)}$.

In the present study, the application of Fluoride varnish was performed and repeated at all treatment appointment to reflect the usual clinical application in children. This application is agreed with that of previous studies, in which repeated FV applications were performed over a short period of time ${ }^{(19)}$. The results of previous studies that agree with our study reported that RI technique is highly effective and minimally invasive and has advantages over other WSLs treatment options. Some authors reported superior reduction in lesion progression for RI accompanied with FV treatment than FV treatment alone. In this study, RI treatment alone was found to be superior to FV treatment immediately after treatment $^{(15)}$.

Most studies reported that there were no side effects with children treated by resin infiltration technique. Other study reported bitter taste after 
removing rubber dam in $(5 \%)$ of cases ${ }^{(16)}$, they considered that a possible overflow of the material in the child mouth. In the present study, only (4.5\%) of cases reported pain in the area of the treatment as we were used Optra gat system instead of rubber dam which usually cause pain and discomfort to children.

\section{CONCLUSIONS}

- Infiltration concept was introduced as an attempt to bridge the gap between "wait and see".

- Resin Infiltration is micro-invasive approach in addition to its applicability and acceptance when used with children.

- A further advantage of infiltration is that unlike fluoride, as it can improve color and the effect appears immediately after treatment.

\section{ACKNOWLEDGMENTS}

We gratefully acknowledge Prof. Dr. Osama El-Shall and Prof. Dr. Mohamed Hassan Mostafa for providing information about their ongoing studies. The authors would like to acknowledge the university for providing the opportunity to perform the research.

\section{RECOMMENDATIONS}

- More experimental data and further clinical research with larger sample sizes and different follow-up periods are needed on ICON resin infiltration compared to other materials.

- Clinical trials should be conducted evaluating children acceptability of the treatment procedure.

\section{CONFLICT OF INTEREST}

The authors declare that there are no conflicts of interest.

\section{FUNDING:}

No funding was received for this study.

\section{REFERENCES}

1. Tellez M, Gomez J, Kaur S, Pretty IA, Ellwood R, Ismail AI. Non-surgical management methods of noncavitated carious lesions. Community Dent Oral Epidemiol. 2013;41:79-96.

2. Correia A, Borges A, Torres C. Color masking prediction of posterior white spot lesions by resin infiltration in vitro. J Dent. 2020; 95: 2-7.

3. Al-Tounsi S. Effect of fluoride varnish, sealants and caries infiltration in reducing the progression of proximal incipient caries in primary teeth. J Den. 2012; 13:115-28

4. Arslan S, Kaplan MH. The effect of resin infiltration on the progression of proximal caries lesions: A randomized clinical trial. Med Princ Pract. 2019;20:243-63

5. Foster Page LA, Beckett D, Ahmadi R, Schwass DR, Leon De La Barra S, Moffat SM, et al. Resin infiltration of caries in primary molars in a community setting: 24 -month randomized controlled trial findings. JDR Clin Transl Res. 2017;2:287-94.

6. Azizi Z. Management of white spot lesions using resin infiltration technique: A Review. Open J Dent Oral Med. 2015;3:1-6.

7. Knösel M, Vogel R, Sandoval P. Infiltration of white-spotlesions and developmental enamel defects. Rev clínica periodoncia, Implantol y Rehabil oral. 2017 Aug;10:101-6.

8. Vaghela P. Resin infiltration of incipient interproximal caries: A 6-month randomized, controlled clinical trial. ProQuest Diss Theses. 2017;71:2026781123.

9. International Caries Detection and Assessment System Coordinating Committee. Rationale and Evidence for the International Caries Detection and Assessment System (ICDAS II). J Istanbul Univ Fac Dent 2015; 49:63-72.

10. Bagher SM, Hegazi FM, Finkelman M, Ramesh A, Gowharji N, Swee G, et al. Radiographic effectiveness of resin infiltration in arresting incipient proximal enamel lesions in primary molars. Pediatr Dent 2018;40:195200.

11. Spagopoulos D, Kotrogianni M, Paximada C. Treatment of white spot lesions due to enamel hypomineralization using the resin infiltration technique. Ital J Dent Med. 2017;5:106-11.

12. Ramos-Gomez F, Cryst YO, Wai Ng M, Tinanoff $\mathrm{N}$, Featherstone JD. Caries risk assessment, prevention and management in pediatric dental care. Pediatric dent 2010; 12:505-17. 
13. Metwally NI, Niazy MA, El-malt MA. Remineralization of early carious lesions using biomimetic self- assembling peptides versus fluoride agent. (In vitro and In vivo study) ADJ-for Girls. 2018;5: 349-58.

14. Featherstone JDB. Dental caries: a dynamic disease process. Aust Dent J 2008; 53: 286-91.

15. Mikhael M, Mendes V, Gomez J, Kaur S, Silva T. Is noncavitated proximal lesion sealing an effective method for caries control in primary and permanent teeth? A systematic review and meta-analysis. J Den 2014; 42: 1217-27.

16. Arslan S, Zorba YO, Atalay MA, Özcan S, Demirbuga $S$, Pala K. Effect of resin infiltration on enamel surface properties and Streptococcus mutans adhesion to artificial enamel lesions. Dent Mater J. 2015;34:25-30.

17. Ten Cate JM, Jongebloed WL, Arends J. Remineralization of artificial enamel lesions in vitro. IV. Influence of fluorides and diphosphonates on short- and long-term reimineralization. Caries Res 2004;15:60-9.

18. Tong LS, Pang MK, Mok NY, King NM, Wei SH. The effects of etching, micro-abrasion, and bleaching on surface enamel. J Dent Res 2006;72:67-71.

19. Autio-Gold JT, Courts F. Assessing the effect of fluoride varnish on early enamel carious lesions in the primary dentition. J Am Dent Assoc 2001;132:1247-53.

20. Bravo M, Baca P, Llodra JC, Osorio E. A 24-month study comparing sealant and fluoride varnish in caries reduction on different permanent first molar surfaces. J Public Health Dent 1997;57:184-6.

21. Garg SA, Chavda SM. Color masking white fluorotic spots by resin infiltration and its quantitation by computerized photographic analysis: A 12-month follow-up study. Oper Dent. 2020; 45:56-64. 